\title{
Formulation factors affecting the isomerization rate of betamethasone-17-valerate in a developmental hydrophilic cream - a HPLC and microscopy based stability study
}

Jonathan Byrne, Anke Wyraz, Trinidad Velasco-Torrijos \& Robert Reinhardt

To cite this article: Jonathan Byrne, Anke Wyraz, Trinidad Velasco-Torrijos \& Robert Reinhardt (2017) Formulation factors affecting the isomerization rate of betamethasone-17-valerate in a developmental hydrophilic cream - a HPLC and microscopy based stability study, Pharmaceutical Development and Technology, 22:4, 537-544, DOI: 10.3109/10837450.2016.1143003

To link to this article: https://doi.org/10.3109/10837450.2016.1143003

曲 Published online: 19 Feb 2016.

Submit your article to this journal ¿

LII Article views: 114

View Crossmark data ¿

Citing articles: 2 View citing articles $\square$ 


\title{
Formulation factors affecting the isomerization rate of betamethasone-17-valerate in a developmental hydrophilic cream - a HPLC and microscopy based stability study
}

\author{
Jonathan Byrne ${ }^{1}$, Anke Wyraz ${ }^{1}$, Trinidad Velasco-Torrijos ${ }^{2}$, and Robert Reinhardt ${ }^{1}$ \\ ${ }^{1}$ Analytical/Formulation Development Department, Mibe GmbH Arzneimittel, Brehna, Germany and 2Department of Chemistry, Maynooth \\ University, Maynooth, Co. Kildare, Ireland
}

\begin{abstract}
The formulation of betamethasone-17-valerate (BV) into topical medicines presents a significant challenge for the formulation chemist. The substance is susceptible to acid and base catalyzed isomerization in aqueous environments, which results in valerate transesterification from carbon 17 to carbon 21 of the steroid ring system. This acyl migration process is of significant clinical importance since the 21-valerate ester possesses only a fraction of the potency of the 17-valerate parent compound. Isomerization of BV should therefore be reduced to a minimum through design of a suitable drug vehicle. In this study, the effect of varying the concentration of several excipient components on the isomerization rate of betamethasone valerate in a model hydrophilic cream has been investigated. These excipients include the emulsifier macrogolstearylether-20/21, the co-emulsifier cetylstearyl alcohol and the thickening agent hydroxyl propyl methylcellulose. Additionally, the influence of $\mathrm{pH}$, the presence of the antioxidant, alpha-tocopherol, as well as the chelating agent, disodium edetate, on the stability of the formulation have been investigated. Trial drug product formulations, which were designed to investigate the influence of the above-mentioned components/parameters were manufactured and their stability was tested according to current ICH Guidelines. The content, purity and crystalline structure of the active substance in these formulations was analyzed by a combination of HPLC and microscopy techniques. The study demonstrates that the rate of isomerization of betamethasone valerate depends significantly on the concentration of emulsifier used in the cream formulation. At higher concentrations of emulsifier the isomerization proceeds rapidly with significant degradation over a period of weeks, whereas at lower concentrations significant degradation may not be observed, even after several years' storage. The influence of the emulsifier has been shown to be independent of the $\mathrm{pH}$ value of the aqueous phase of the cream. These findings have not been reported in previous literature reports on this topic, which have tended to focus on the influence of $\mathrm{pH}$. The results are likely to be of interest to pharmaceutical chemists working on the formulation of glucocorticoids as well as to local- and hospital pharmacists who carry out the practice of dilution of proprietary corticoid preparations, where the choice of diluent is likely to be critical for ensuring the stability of the diluted product.
\end{abstract}

\author{
Keywords \\ Chromatography, corticosteroid, emulsifier, \\ isomerization, $\mathrm{pH}$, topical formulation
}

History

Received 16 November 2015

Revised 21 December 2015

Accepted 3 January 2016

Published online 12 February 2016

\section{Introduction}

Betamethasone is a potent, synthetic corticosteroid with antiinflammatory properties, which is employed in the treatment of inflammatory skin disorders such as eczema and psoriasis. Since only $12-14 \%$ of the administered dose of betamethasone is absorbed when applied topically ${ }^{1}$, it must be administered in the form of one of its esters, such as betamethasone-17-valerate (BV) or betamethasone-17,21-dipropionate. The esterified forms generally have increased lipophilic nature allowing for better penetration of the drug molecule across the lipid membranes of

Address for correspondence: Jonathan Byrne, Analytical/Formulation Development Department, Mibe $\mathrm{GmbH}$ Arzneimittel, Brehna 06796, Germany. Tel: +49 34954/247414. E-mail: jonathan.byrne@mibegmbh.de the $\operatorname{skin}^{2,3}$, thus enhancing the local bioavailability of the substance. BV is one of the more potent and commonly used esters of betamethasone and is available in several marketed products for topical use, such as Betnovate ${ }^{\circledR}$ Cream (Glaxo, Brentford, UK) for monotherapy or together with fusidic acid in Fusibet $^{\circledR}$ Cream (Leo Laboratories, Ballerup, Denmark) for combinational therapy of inflammatory skin-conditions with secondary infections.

The formulation of $\mathrm{BV}$ into a suitably stable vehicle is not without problems. The substance is susceptible to an acid and base catalyzed isomerization in aqueous and semi-solid formulations resulting in an acyl group migration from position $\mathrm{C} 17$ to the more stable $\mathrm{C} 21$ position of the steroid ring system ${ }^{4}$ (Figure 1). This isomerization process is of significant clinical relevance since betamethasone-21-valerate demonstrates only $\approx 1 / 15$ th of the potency of its 17 -valerate counterpart ${ }^{5}$. In a highly regulated 

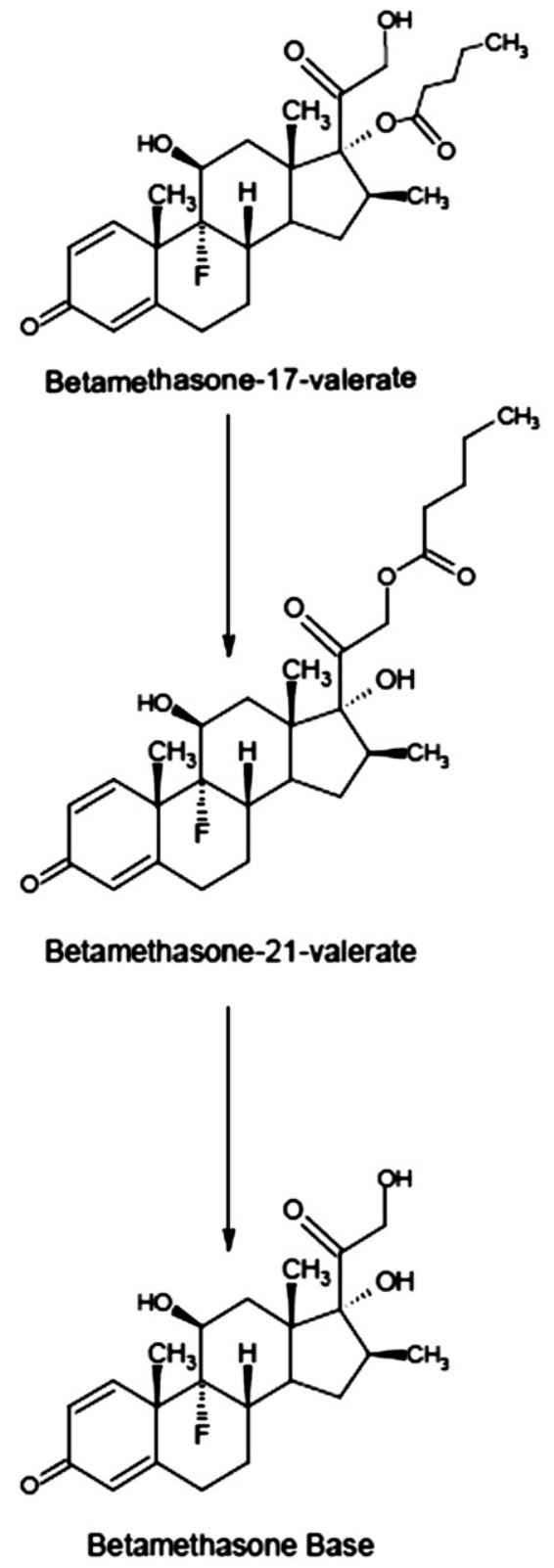

Figure 1. Schematic of isomerization followed by hydrolysis of BV.

environment, such as the pharmaceutical industry, the stability of pharmaceutical preparations is ensured through extensive analytical testing. The data generated through this rigorous testing is used as a basis for justifying the shelf-life of a pharmaceutical product. In contrast, in a clinical setting, such as in a hospital pharmacy, physicians often like to prescribe dilutions of topical corticosteroids, e.g. 1:4 or 1:10 dilutions are typically performed with available diluents. The purpose of diluting corticosteroid preparations is usually to reduce the side-effects associated with their use, such as skin atrophy and pigmentation loss ${ }^{6}$. The practice of dilution of marketed corticosteroids appears in principle to be justified, since numerous studies have shown that the relationship between concentration and activity of topically applied glucocorticoids is not linear ${ }^{6,7}$, with much weaker dilutions often demonstrating similar activity to the undiluted product in vasoconstriction assays $^{8-11}$. However, since in many cases the diluted product is not analyzed and little is known regarding its stability, there may be potential issues regarding safety and efficacy of the active substances in such dilutions.

Previously published studies on this topic have demonstrated that the dilution of semi-solid formulations containing $\mathrm{BV}$ can affect the stability of the drug substance. It has been shown that the choice of diluent plays a crucial role with regard to the isomerization process. For example, 1:4 dilutions of Betnovate ${ }^{\circledR}$ in Unguentum Merck and white soft paraffin were stable over 12 months ${ }^{10,11}$, whereas dilutions in Emulsifying Ointment showed $50 \%$ degradation within $4.2 \mathrm{~h}^{12}$. Many of the published studies offered little explanation as to why the diluent was influencing the isomerization rate. In most cases, the increased rate of isomerization was attributed to an increased $\mathrm{pH}$ of the diluted preparation, without consideration of other factors. Boonsaner et al. ${ }^{8}$ studied the stability of BV in Beeler's Basis and Cold Cream ${ }^{\circledR}$. They came to the conclusion that the degradation rate cannot be solely dependent on the $\mathrm{pH}$ alone and that other unknown factors must be involved. It was shown that preparations with similar $\mathrm{pH}$ values can show very different stabilities but no evidence was provided as to what these unknown factors may have been.

In this study, it has been demonstrated that the concentration of the primary emulsifier in a hydrophilic cream formulation plays a crucial role with regard to the isomerization rate of $\mathrm{BV}$. The influence of the concentration of the emulsifiers macrogolstearylether-20/21 (S-20/S-21) as well as the co-emulsifier cetylstearyl alcohol (CSO) and thickening agent hydroxyl propyl methylcellulose (HPMC) on the isomerization rate in experimental cream formulations has been investigated. Additionally, the influence of $\mathrm{pH}$, the presence of the antioxidant, alpha-tocopherol, as well as the chelating agent, disodium edetate, on the stability of the formulation have been investigated. The aim of these studies was to develop a stable pharmaceutical vehicle for the effective delivery of betamethasone valerate and fusidic acid.

The content, purity and crystalline structure of the drug substance in each of the formulations has been analyzed by a combination of HPLC and microscopic techniques. The HPLC analysis was performed using two novel procedures, which were specifically developed for this task ${ }^{13,14}$. This study is the first to demonstrate that the concentration of emulsifier in the finished product is of critical importance with regard to the isomerization of $\mathrm{BV}$ and thus its stability in semi-solid formulations.

\section{Materials and methods}

\section{Reagents}

Gradient grade methanol and acetonitrile were purchased from VWR International GmbH (Darmstadt, Germany). Purified water was obtained from the in-house purification system at Mibe GmbH Arzneimittel (Brehna, Germany). Phosphoric acid (85\% $\mathrm{m} / \mathrm{m}$, analysis grade, $d=1.71 \mathrm{~g} / \mathrm{ml}$ ) was purchased from Merck (Darmstadt, Germany). All cream samples were provided by Mibe GmbH Arzneimittel.

A description of the function and quality of the APIs and excipients used in the manufacture of the cream is described in the table.

\begin{tabular}{lll}
\hline Ingredient & Function & Quality \\
\hline $\begin{array}{l}\text { Fusidic acid hemihydrate } \\
\text { Betamethasone-17-valerate }\end{array}$ & $\begin{array}{l}\text { drug substance } \\
\text { drug substance }\end{array}$ & Ph. Eur. \\
Macrogol stearyl ether 20 & emulsifier & Ph. Eur. \\
& & In-house \\
Cetylstearylalcohol & co-emulsifier & Ph. Eur. \\
Paraffin, liquid & ingredient of the oil phase & Ph. Eur. \\
Paraffin, white soft & ingredient of the oil phase & Ph. Eur. \\
Hypromellose & thickener & Ph. Eur. \\
Citric acidmonohydrate & pH modifying agent & Ph. Eur. \\
Methyl parahydroxybenzoate & preservative & Ph. Eur. \\
\hline
\end{tabular}




\begin{tabular}{lll}
\hline Ingredient & Function & Quality \\
\hline Propylparahydroxybenzoate & preservative & Ph. Eur. \\
Potassium sorbate & preservative & Ph. Eur. \\
Water, purified & solvent & Ph. Eur. \\
\hline
\end{tabular}

\section{Instrumentation}

HPLC experiments were performed with Shimadzu Prominence HPLC Systems (Shimadzu, Tokyo, Japan). The systems were equipped with a binary pump (LC-20AD), a temperaturecontrolled auto-sampler (SIL-20AC $\mathrm{HT}_{\mathrm{HT}}$ ), a temperature-controlled column compartment (CTO-20AC) as well as an on-line degasser (DGU-20A 5 ). A (SPD-M20A) DAD detector was employed. The software packages used were SHIMADZU LCsolution version 1.24 SP1 and SHIMADZU Class-VP version 6.14 SP2A. Stability studies were performed in Binder KBF 720 stability chambers (Binder Inc., Tuttlingen, Germany). Microscopy experiments were performed with a Nikon Eclipse LV100 polarizing microscope (Nikon, Tokyo, Japan) equipped with diascopic and episcopic illuminators (LV-UEPI2), a polarizer, a CFIUW $10 \times$ binocular, CFI LU Plan Fluor Epi P $5 \times, 10 \times, 20 \times, 40 \times$ and $100 \times$ objectives and a DS-Fi2 camera unit. Analysis of the micrographs was performed using NIS-Elements AR software version v.4.2. The HPLC conditions and sample preparation procedure are described in the literature ${ }^{13,14}$.

\section{Experimental procedures}

\section{Solubility in paraffin}

BV (60.0 mg) was weighed into a 50-ml centrifuge tube and $15 \mathrm{ml}$ of liquid paraffin was added. The resulting suspension was shaken in a water-bath at $65^{\circ} \mathrm{C}$ for $4 \mathrm{~h}$.

\section{Solubility in aqueous solutions of emulsifier}

Ten milligrams of BV was weighed into a 50-ml centrifuge tube and mixed with $25 \mathrm{ml}$ of aqueous solutions of S-21 of varying concentration over the range $0.0-1.0 \%$ of the emulsifier. The mixtures were shaken for $4 \mathrm{~h}$ in a water-bath at $65^{\circ} \mathrm{C}$ and allowed to cool to room temperature. The $\mathrm{pH}$ of each mixture was recorded and the mixtures were filtered through a $0.45-\mu \mathrm{m}$ regenerated cellulose syringe filter into a vial before HPLC analysis.

\section{Preparation of cream samples}

Cream formulations were manufactured in several steps. In the first step, macrogolstearylether-20/21 (S-20/S-21), CSO, liquid paraffin and white soft paraffin were melted by heating in a suitable vessel under stirring. Next, the aqueous phase was prepared by dissolving the required quantities of the preservative compounds potassium sorbate as well as methyl- and propyl paraben in purified water. Both the aqueous and oil phases were mixed and homogenized by stirring under heating for an appropriate length of time forming a cream base. A gel phase was then prepared by dissolving the required quantities of citric acid monohydrate and HPMC in purified water by stirring at elevated temperature. The gel phase was added to the cream base and the homogenization procedure was repeated. Finally, an active substance suspension was prepared by suspending the API in liquid paraffin. This suspension was then homogenized and added to the cream at elevated temperature. The cream was allowed to cool before being homogenized for a final time.

\section{Results}

\section{Initial trial batches}

Initially, a number of trial formulations were prepared with varying quantities of selected excipients. The proportions of S-20, CSO and hydroxylpropyl methylcellulose (HPMC) contained in these formulations are provided in Table 1. For the purposes of this study, the batches have been labeled as variants A, B, C and D. Variant A also contained $0.1 \%$ of the antioxidant alphatocopherol, which was added to investigate if oxidative processes were likely to affect the stability of the drug substance. The quantities of all other ingredients of the cream as well as the manufacturing procedure and equipment were similar for all batches. In each case, the $\mathrm{pH}$ was adjusted to $5.0 \pm 0.2$ and the active pharmaceutical ingredient was present in suspended form in the cream matrix.

The stability of each of the trial batches was determined after storage for 1 month under real-time $\left(25^{\circ} \mathrm{C} / 60 \% \mathrm{RH}\right)$ and accelerated $\left(40^{\circ} \mathrm{C} / 75 \% \mathrm{RH}\right)$ conditions (see Figure 2 for a representative example chromatogram). The results indicated that variant $\mathrm{D}$ was markedly more stable than any of the other variants with regard to the isomerization rate (Figure 3a). Variants A, B and $\mathrm{C}$ contained approximately $3-4 \%$ of the isomerization product betamethasone-21-valerate after storage for 1 month at $25^{\circ} \mathrm{C} / 60 \%$ $\mathrm{RH}$. This level of degradation was considered unacceptable since this quantity of impurity would normally be expected to be present after 24-36 months storage in a comparable pharmaceutical product. Furthermore, current guidelines ${ }^{15,16}$ require that the content of the drug substance should lie between $95 \%$ and $105 \%$ or, if justified, between $90 \%$ and $105 \%$ of the label claim throughout the shelf-life of the product. The above results indicated that the API would have been completely degraded after 36 months, if the isomerization had continued at a linear rate.

The data also seemed to indicate that reducing the quantity of HPMC from $1.00 \%$ to $0.50 \%$ may slow the rate of isomerization, whereas, in contrast, increasing the quantity of HPMC from $1.00 \%$ to $2.00 \%$ did not affect the stability of BV. Decreasing the quantity of S-20 to $3.65 \%$ in combination with an increase of CSO to $6.35 \%$ may also influence the rate of isomerization. As evident by a comparison of the data from variants $\mathrm{A}$ and $\mathrm{B}$, the addition of $0.1 \%$ alpha-tocopherol to the formulation has no effect on the rate of isomerization. It was therefore concluded that oxidative

Table 1. The percentages of S-20, CSO and HPMC in the first trial variants A, B, C and D.

\begin{tabular}{|c|c|c|c|c|c|}
\hline & & Variant A & Variant B & Variant $\mathrm{C}$ & Variant D \\
\hline Article & Description & Proportion in mass $\%$ & Proportion in mass $\%$ & Proportion in mass $\%$ & Proportion in mass $\%$ \\
\hline 1 & Macrogolstearylether-20 (S-20) & 5.00 & 5.00 & 5.00 & 3.65 \\
\hline 3 & Hypromellose (HPMC) & 1.00 & 1.00 & 2.00 & 0.50 \\
\hline 4 & $\alpha$-Tocopherol & $0.1 \%$ & - & - & - \\
\hline
\end{tabular}


Figure 2. Example chromatogram from the purity test of a degraded cream sample analyzed after stressing under the conditions of $40{ }^{\circ} \mathrm{C} / 75 \% \mathrm{RH}$ at $\mathrm{pH} 7$ for 2 months. The sample demonstrated significant degradation. HPLC conditions as provided in Table 1. Peak 3 was not present in this particular sample. 1, Betamethasone; 2, 24,25-dihydro24,25-dihydroxyfusidic acid; 3, 24,25-dihydro-24,25-dihydroxyfusidic acid-21,25-lactone; 4 , betamethasone-17-valerate; $5,(24 R)$ 24,25-dihydro-24,25-dihydroxyfusidic acid 21,24-lactone; 6, 26-hydroxyfusidic acid; 7, (24S)-24,25-dihydro-24,25-dihydroxyfusidic acid 21,24-lactone; 8, Betamethasone-21valerate; 9, 26-oxofusidic acid; 10, 3-didehydrofusidic acid; 11, 11-didehydrofusidic acid; 12, 16-epideacetylfusidic acid; 13, fusidic acid; 14, 16-epideacetylfusidic acid21,16-lactone; 15 , deacetylfusidic acid21,16-lactone; 16, 11-deoxyfusidic acid. $\mathrm{U}$, unknown impurity.

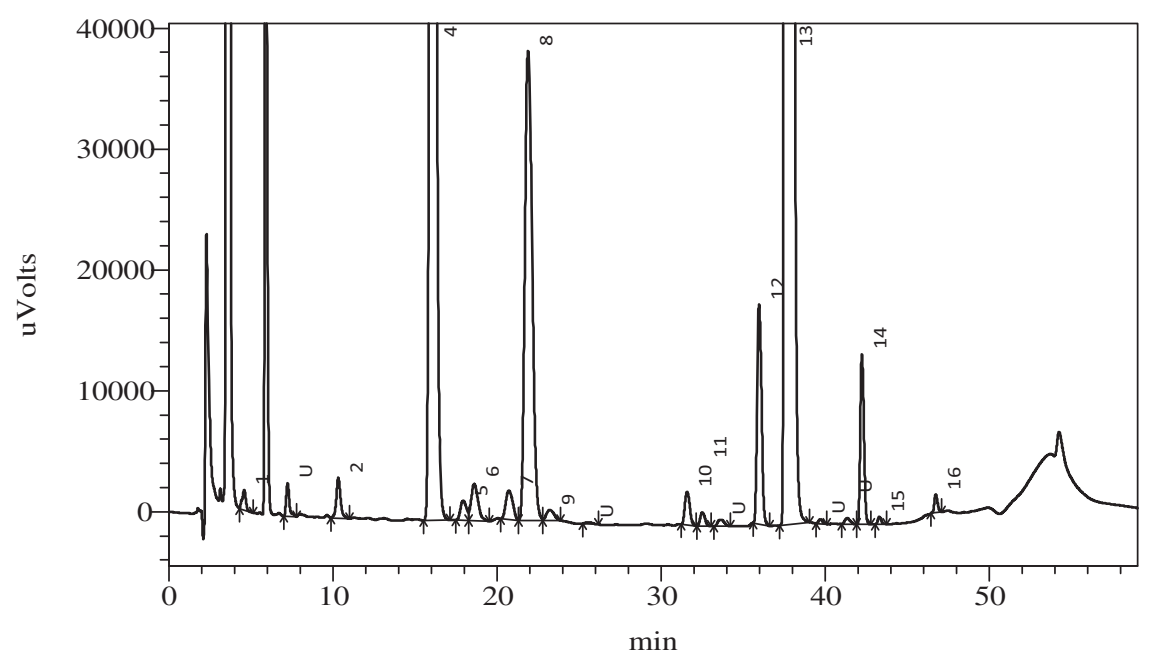

(a)

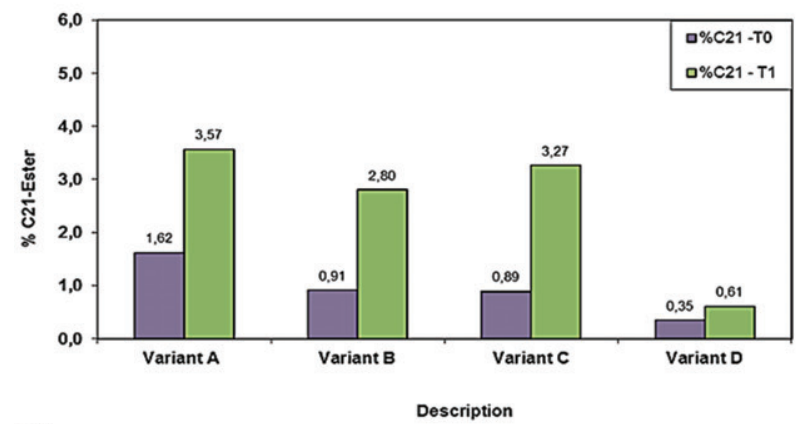

(c)

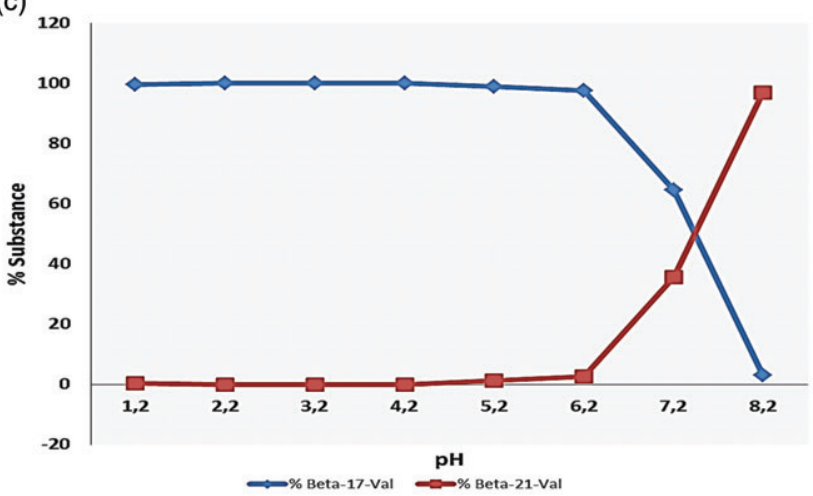

(b) Isomerisation of betamethasone-17-valerate

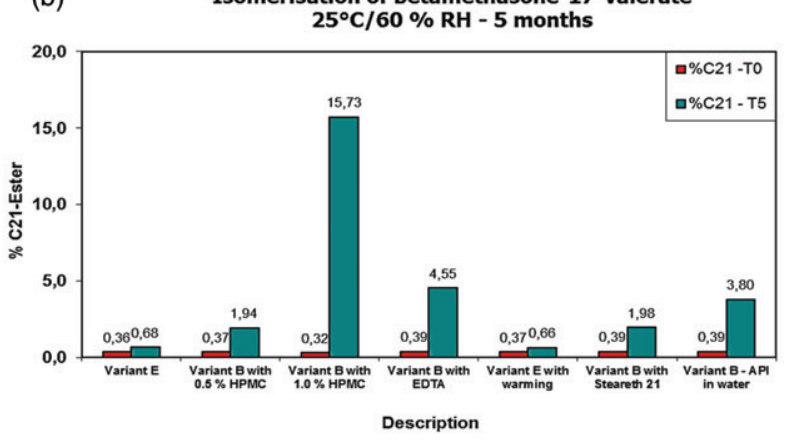

(d)

Solubility and Stability of Betamethasone-17-valerate in an aqueous Solution of Steareth 21

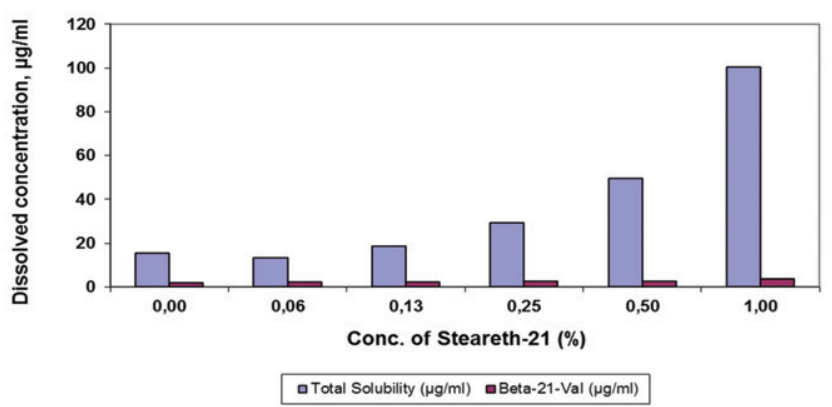

Figure 3. (a) Initial trial batches - content of betamethasone-21-valerate after storage for 1 month at $25^{\circ} \mathrm{C} / 60 \%$ RH. (b) Optimized formulations content of betamethasone-21-valerate after storage for 5 months at $25^{\circ} \mathrm{C} / 60 \% \mathrm{RH}$. (c) Influence of pH on the isomerization rate of $\mathrm{BV}$ in aqueous solution within the $\mathrm{pH}$ range 1.2-8.2 after $48 \mathrm{~h}$. (d) Influence of the concentration of steareth-21 on the solubility of BV in an aqueous medium.

degradation processes are unlikely to play a role in the observed degradation of BV in the investigated hydrophilic formulation.

\section{Optimization - round I}

Despite the observed trend, the stability of the first trial batches proved to be unacceptable and it was therefore necessary to optimize the formulation. The compositions of the optimized trial formulations are provided in Table 2. These formulations were subjected to stability studies in a similar fashion to the initial batches and tested by HPLC. The 5 months real time data presented in Figure 3(b) demonstrate that increasing the content of HPMC from $0.50 \%$ to $1.00 \%$ accelerates the rate of isomerization. In contrast, a reduction of the quantity of Steareth-20 from $5.00 \%$ (variant B) to $2.14 \%$ (variant E) coupled with an increase of the quantity of CSO from $5.00 \%$ (variant B) to $7.86 \%$ (variant E) appeared to slow the rate of isomerization. Replacing S-20 with macrogolstearylether-21 (S-21) may slow the rate of isomerization, whereas suspending the API in the aqueous phase of the cream, as opposed to the oil phase, does not stabilize the formulation.

HPMC is present in the formulation as a thickening agent, which should not affect the stability of BV. However, according to the Technical Handbook for METHOCEL Cellulose Ethers from 
Table 2. The percentages of S-20, CSO and HPMC in the optimized versions of variants B and E.

\begin{tabular}{|c|c|c|c|c|c|}
\hline & & Variant E & Variant E (with warming) & Variant B $(0.5 \%$ HPMC $)$ & Var. B (1.0\% HPMC) \\
\hline 1 & Macrogolstearylether-20 (S-20) & 2.1 & 2.1 & 5.0 & 5.0 \\
\hline \multirow[t]{2}{*}{3} & Hypromellose (HPMC) & 0.5 & 0.5 & 0.5 & 1.0 \\
\hline & & Variant $\mathrm{B}\left(0.1 \% \mathrm{Na}_{2} \mathrm{EDTA}\right)$ & Variant B (with S-21) & Variant $\mathrm{B}\left(\mathrm{API}\right.$ in $\left.\mathrm{H}_{2} \mathrm{O}\right)$ & \\
\hline 1 & $\begin{array}{l}\text { Macrogolstearylether-20 “or" } \\
\quad-21(\mathrm{~S}-20 / \mathrm{S}-21)\end{array}$ & 5.0 & 5.0 & 5.0 & \\
\hline 2 & Cetylstearyl alcohol (CSO) & 5.0 & 5.0 & 5.0 & \\
\hline 3 & Hypromellose (HPMC) & 1.0 & 1.0 & 2.0 & \\
\hline
\end{tabular}

the DOW chemical company, Methocel (HPMC) also possesses emulsifying properties and can act as a surfactant in solution. It was therefore considered theoretically possible that HPMC may help to increase the solubility of BV in the aqueous phase leading to an increased rate of isomerization. After investigation the increased rate of isomerization was found to be due to this variant having a $\mathrm{pH}$ value of 6.0 (in contrast to the other variants which had $\mathrm{pH}$ values of $5.0 \pm 0.2$ ).

It was known from the studies of Bundgaard and Hansen ${ }^{17}$ that the kinetics of the transesterification of $\mathrm{BV}$ to the 21-valerate ester in aqueous solution is $\mathrm{pH}$ dependent. It was therefore considered likely that the shift in $\mathrm{pH}$ of the cream from 5 to 6 was responsible for the increase of the isomerization rate. When the $\mathrm{pH}$ of the cream was kept constant, the concentration of HPMC had no influence on the isomerization rate. Experiments in aqueous media later showed that the $\mathrm{pH}$ increases with increasing HPMC concentration.

Literature studies indicate that the presence of trace metals in pharmaceutical preparations can cause degradation of glucocorticoids $^{18,19}$. For this reason, the influence of the presence of the chelating agent disodium edetate on the degradation rate of BV was investigated. The results did not indicate an improvement as compared to variant B. It was concluded that metal ions are not a determining factor with regard to the isomerization rate of $\mathrm{BV}$.

The suspension of the API in the aqueous phase did not influence the isomerization rate, indicating that the presence of water alone is not sufficient to initiate the isomerization process. This was perhaps to be expected since BV is practically insoluble in water ${ }^{20}$. This observation, however, suggested that the API must be in solution for the isomerization to take place and, furthermore, that some component of the cream must be enhancing the solubility of BV in the aqueous phase. Since it had been observed that a reduction of the concentration of S-20 may lead to an increased stability, it was suspected that the emulsifier was aiding the dissolution of the suspended BV crystals into the aqueous phase of the cream.

Replacing S-20 with S-21 appeared to decrease the isomerization rate in the above experiment. S-20 and S-21 differ in the number of oxyethylene $\left(-\mathrm{OC}_{2} \mathrm{H}_{5}\right)$ groups attached to the stearic acid chain. Both substances have very similar HLB (hydrohiliclipophilic-balance) values (15.3 and 15.7, respectively, according to the manufacturer) indicating that they are likely to show very similar solubilities in aqueous solution. It would therefore be expected that both substances should influence the stability of the cream formulation to a similar extent. Repeat experiments could not confirm the initial results and it was finally concluded that there is no major difference between these substances with regard to their influence on the isomerization process. However, due to commercial and regulatory considerations it was decided to employ S-21 in all further experiments.

\section{Optimization - round II}

The results of the initial- and optimization experiments demonstrated that reducing the content of S-20 from 5.00\% (variants A, $\mathrm{B}$ and $\mathrm{C}$ ) to $3.65 \%$ (variant D) and further to $2.14 \%$ (variant $\mathrm{E}$ ) significantly slowed the rate of isomerization. Consequently, further trial batches were produced containing less S-21 and with varying quantities of CSO. The goal was to find the optimum concentration of these components which would provide the lowest rate of isomerization without compromising the physical stability of the cream. The proportions of liquid paraffin and vaseline in the cream were also adjusted with the aim of improving the physical stability of the preparation. The accelerated stability studies demonstrated that maximum physical- and chemical stability was achieved with $1.5 \% \mathrm{~S}-21$ and $5.5 \%$ CSO (Variant F). Further reducing the S-21 concentration to $1.00 \%$ did not appear to bring further improvement. Under real time conditions variant $\mathrm{F}$ showed no apparent increase of betamethasone-21-valerate content after 1 month and $<3 \%$ increase over a period of 30 months storage.

Optimization of the concentrations of S-21 and CSO resulted in an increased chemical stability of BV, while optimization of the proportions of vaseline and liquid paraffin lead to an increased physical stability of the cream preparation. In order to demonstrate definitively that the reduction of the concentrations of the emulsifier had lead to the increased stability of BV, and not other parameters, for example the adjustment of the vaseline and paraffin quantities, the concentration of S-21 in variant $F$ was increased to the initial concentration present in variants $\mathrm{A}, \mathrm{B}$ and C, i.e. $5.00 \%$. The relative proportions of vaseline, paraffin and $\mathrm{CSO}$, as well as all other excipients remained equivalent to the optimized variant $\mathrm{F}$. The results demonstrated clearly that an increase of the concentration of the primary emulsifier, S-21, leads to an increase of the isomerization rate.

\section{Effect of $\mathrm{pH}$ on the isomerization rate of $\mathrm{BV}$}

The effect of $\mathrm{pH}$ on the isomerization rate of $\mathrm{BV}$ was investigated in both aqueous solution using appropriate buffers and in the cream preparation itself. As shown in Figure 3(c) the API appears stable in aqueous solution at $\mathrm{pH}$ values between 2.2 and 4.2 with an optimum at $\approx \mathrm{pH} 3.2$. This is in good agreement with the reported literature ${ }^{21}$. At $\mathrm{pH}$ values above 4.2 the rate of isomerization increases gradually, then increasing very rapidly 
above $\mathrm{pH}$ 6.2. Complete isomerization of $\mathrm{BV}$ was observed after $48 \mathrm{~h}$ at $\mathrm{pH} 8.2$.

The situation is similar in the cream preparation. At $\mathrm{pH}$ values between 2.7 and 5.3 the drug substance was relatively stable, although some degradation still occurred. At higher $\mathrm{pH}$ values, e.g. $>6$, the isomerization rate increases very rapidly giving rise to complete isomerization within 1 month at $\mathrm{pH} 8$ and $40^{\circ} \mathrm{C} / 75 \%$ RH. A pH range of between 4.5 and 5.5 was chosen for the cream since this range is a compromise between skin tolerability and drug stability. Lower $\mathrm{pH}$ values are generally considered too acidic for topical treatment and higher $\mathrm{pH}$ values would compromise the stability of the API.

\section{Experiments in aqueous solution}

During manufacture of the cream preparation, BV is suspended in liquid paraffin before being added to the rest of the ingredients during the last stage of manufacture. In order to confirm that the isomerization only occurs when an aqueous medium is present, the stability of $\mathrm{BV}$ in liquid paraffin was investigated (see Experimental Procedures, Section "Solubility in paraffin"). The conditions employed were known to give rise to isomerization in aqueous solution. No isomerization was observed in liquid paraffin, indicating that water is necessary for the isomerization to take place. Since the API crystals are initially suspended in the cream, the BV must dissolve into the aqueous (continuous) phase of the $\mathrm{O} / \mathrm{W}$ emulsion in order for the isomerization to occur. This hypothesis was further investigated by experimentation in aqueous solution.

\section{Effect of $S-21$ concentration on the solubility of $B V$ in aqueous medium}

In order to examine the influence of the concentration of S-21 on the solubility and isomerization rate of $\mathrm{BV}$ in aqueous solution, solubility measurements were carried out (see Experimental Procedures, Section "Solubility in aqueous solutions of emulsifier'). It was found that all solutions had a $\mathrm{pH}$ of $5.3 \pm 0.2$. Figure 3(d) shows how the solubility of BV increases in aqueous solution with increasing concentration of S-21. In contrast, the rate of isomerization does not increase proportionally because of the relatively favorable $\mathrm{pH}$ of 5.3 which prevents a rapid isomerization (see Section "Solubility in aqueous solutions of emulsifier'). It was concluded that S-21 increases the solubility of $\mathrm{BV}$ in aqueous media. In the drug product formulation the BV crystals are also exposed to both the water phase and to the emulsifier and it was suspected that in this case the increased emulsifier concentration also leads to an increased solubility of BV.

\section{Effect of variation of both $\mathrm{S}-21$ concentration and $\mathrm{pH}$}

The effect of simultaneously varying both S-21 concentration and $\mathrm{pH}$ was investigated by preparing samples in the same fashion as described in Section "Effect of S-21 concentration on the solubility of BV in aqueous medium', the only difference being that appropriate buffers were employed over the $\mathrm{pH}$ range 3.2-7.2 (Figure 4). At higher $\mathrm{pH}$ values, e.g. 7, and low concentrations of $\mathrm{S}-21$, the rate of isomerization remains low, indicating that the BV must be solubilized into solution before the isomerization can take place. However, at lower $\mathrm{pH}$ values, e.g. $\mathrm{pH} 3$, and higher concentrations of S-21, the rate of isomerization remains low, indicating that the isomerization only takes place when the $\mathrm{pH}$ conditions allow. The results indicate that the BV must first be solubilized into the aqueous phase where the rate of isomerization is then $\mathrm{pH}$ dependent. In the cream formulation, this means that higher concentrations of emulsifier more rapidly solubilize the

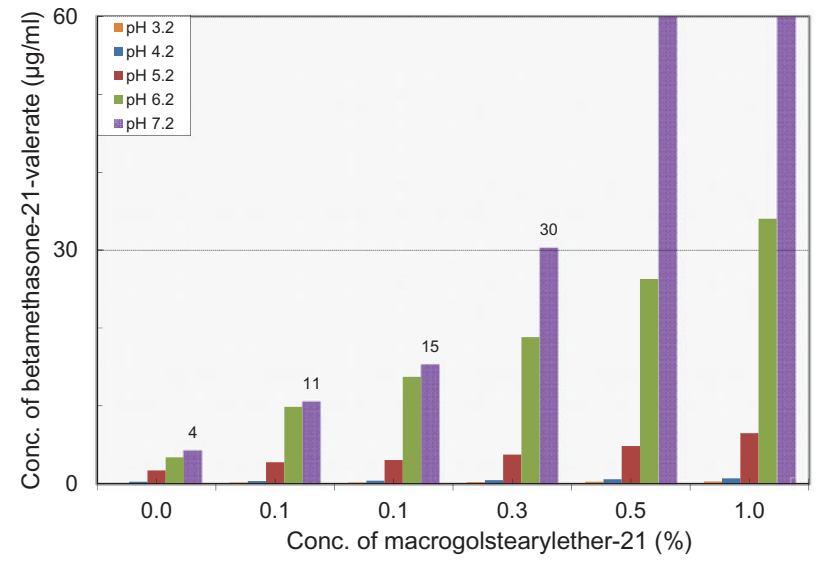

Figure 4. Effect of $\mathrm{pH}$ and emulsifier concentration on the isomerization rate of $\mathrm{BV}(\mathrm{pH} 3.2-7.2)$.

BV thus increasing the overall concentration of drug substance present in the aqueous phase.

\section{Microscopy}

The effect of changing the concentration of emulsifier on the isomerization rate was also examined by microscopy. This technique allows for the direct observation of the suspended BV crystals in real time. However, the BV employed in the cream formulation was micronized (particle size distribution of $90 \%<5 \mu \mathrm{m}$ ) which made the observation of the crystals difficult in such a complex sample matrix. This problem was overcome by employing non-micronized BV with a much larger particle size $(90 \%<110 \mu \mathrm{m})$, thus making the observation of any potential dissolution phenomena much easier. One batch of each of Variant $\mathrm{B}$ and Variant $\mathrm{F}$ was prepared with non-micronized $\mathrm{BV}$ and placed on stability for 1 year under accelerated conditions $\left(40^{\circ} \mathrm{C} / 75 \%\right.$ $\mathrm{RH})$. The microscopic profiles and chemical stability of the creams were analyzed after 1, 2, 3, 6, 9 and 12 months.

The micrographs presented in Figure 5 demonstrate that the BV crystals in Variant B (with 5\% S-21) disintegrate and dissolve over time. After 12 months storage at $40{ }^{\circ} \mathrm{C} / 75 \% \mathrm{RH}$ there are no visible crystals remaining in the cream. The data from the chemical analysis also indicate that the BV is rapidly degrading under these conditions. In contrast, the BV crystals in Variant $\mathrm{F}$ remain largely unchanged, a well-defined crystal structure being present throughout the entire study. The isomerization rate of BV is also much slower in variant $\mathrm{F}$ than in variant $\mathrm{B}$, as confirmed by the chemical data. This study further supports the hypothesis that increasing the concentration of emulsifier leads to a more rapid dissolution of the suspended crystals.

\section{Discussion and conclusions}

The current study has demonstrated that the $\mathrm{pH}$ of a topical vehicle is not the only factor to be considered when formulating $\mathrm{BV}$. The stability of BV in a given preparation may be influenced by adjusting the concentration of the emulsifier to such a degree that either practically no isomerization occurs over the shelf-life, or alternatively, that complete isomerization occurs within a short period of time. An increased concentration of macrogolstearylether has been shown to enhance the solubility and isomerization rate of $\mathrm{BV}$ in the aqueous phase of a hydrophilic cream. Maximum chemical stability was achieved at a concentration $<1.5 \%$ (w/w) of this substance in the preparation. The data suggest that the co-emulsifier CSO does not play a significant role in the solubilization/isomerization process. 


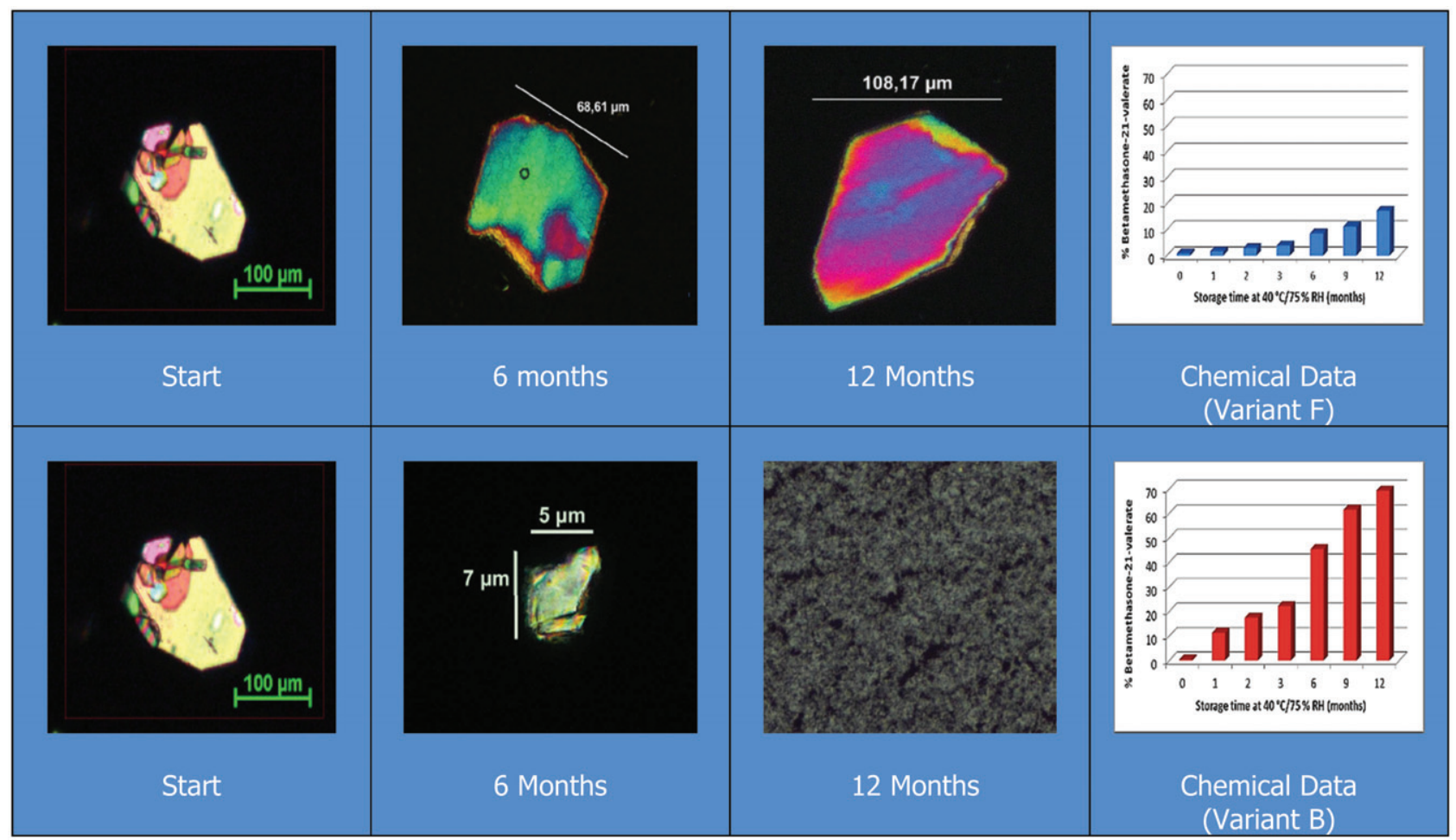

Figure 5. Micrographs of BV crystals in variants $\mathrm{B}$ and $\mathrm{F}$ after storage at $40^{\circ} \mathrm{C} / 75 \% \mathrm{RH}$.

Figure 6. Schematic of the effect of $\mathrm{pH}$ and emulsifier concentration on the isomerization rate of $\mathrm{BV}$ in a hydrophilic cream.

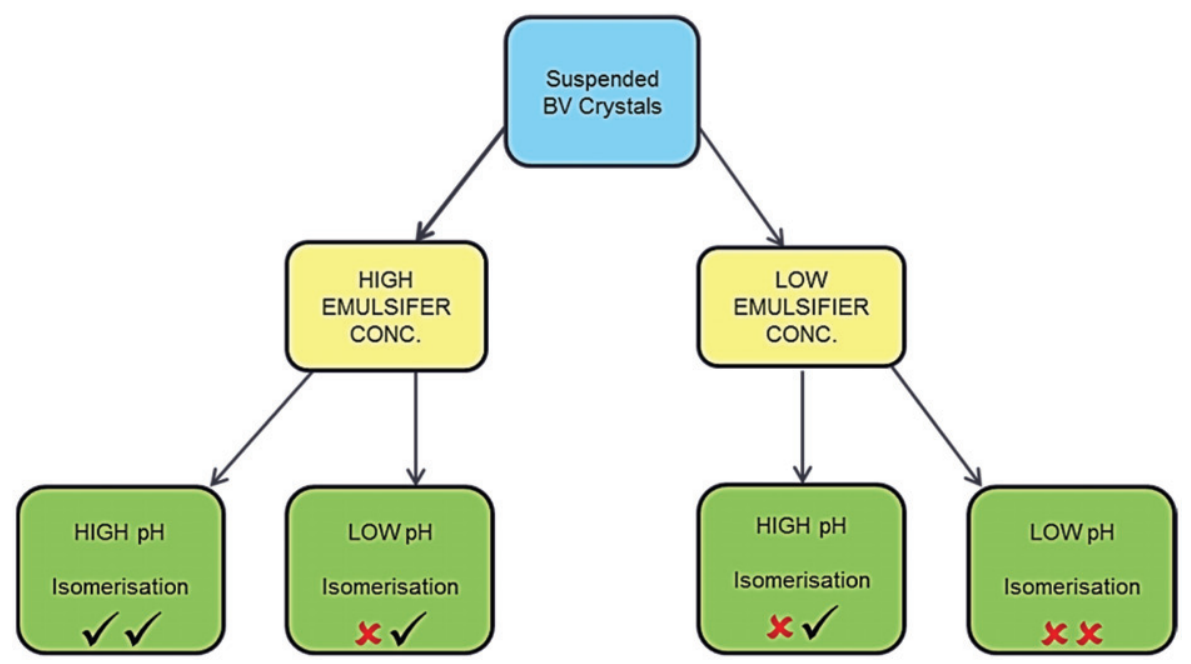

Overall, the factors influencing the rate of isomerization in a hydrophilic cream in which BV is suspended can be summarized as follows (Figure 6):

- Water is necessary for the isomerization to take place. Transesterification will not occur in a non-aqueous environment, e.g. fatty ointment.

- At high concentrations of emulsifier (e.g. 5\%) and higher $\mathrm{pH}$ values (e.g. $>6$ ) there is a rapid rate of isomerization.

- At high concentrations of emulsifier (e.g. 5\%) and lower $\mathrm{pH}$ values (e.g. $<4)$ there is a moderate rate of isomerization, which is $\mathrm{pH}$ dependent.

- At low concentrations of emulsifier (e.g. 1\%) and higher pH values (e.g. $>6$ ) there is a moderate rate of isomerization, which appears to be mainly dependent on the solubility of the API in the aqueous phase.
- At low concentrations of emulsifier (e.g. 1\%) and lower pH values (e.g. <4) there is a slow rate of isomerization since the concentration of $\mathrm{BV}$ in the aqueous phase is very low and the quantity which is dissolved isomerizes at a slow rate due to the low $\mathrm{pH}$.

In order to optimize the stability of BV in a hydrophilic cream suspension, both the concentration of emulsifier and the $\mathrm{pH}$ value should be kept as low as possible. It seems reasonable to hypothesize that the instabilities observed on dilution of $\mathrm{BV}$ creams with emulsifying ointment and other emulsifier containing diluents in previous studies ${ }^{11,12}$ may have been due to an increased solubility of $\mathrm{BV}$ in the aqueous phase. This study demonstrates that the choice and concentration of emulsifier chosen for a topical vehicle containing $\mathrm{BV}$ is likely to be of critical importance when developing a formulation. Equally, the 
diluent used for diluting proprietary marketed corticosteroid preparations must be chosen with care, taking not only $\mathrm{pH}$, but also emulsifier concentration into account. Based on the observed mechanism, it seems plausible that the findings are also relevant for other topical glucocorticoids esterified at the C17 position, although this remains to be confirmed.

\section{Declaration of interest}

The authors have no conflicts of interest to declare.

\section{References}

1. Kommentar zur Ph. Eur. 6.0, Betamethasone 6.0/0312, 30. LfG; 2008.

2. Svensson CK. Biotransformation of drugs in human skin. Drug Metab Dispos 2009;37:247-253.

3. Chan SY, Li Wan Po A. Prodrugs for dermal delivery. Int J Pharm 1989;55:1-16.

4. Yip YW, Li Wan Po A. The stability of betamethasone-17-valerate in semi-solid bases. J Pharm Pharmacol 1979;31:400-402.

5. Middleton KR, Haines-Nutt RF. The stability of diluted betamethasone 17-valerate topical preparations. Hospital pharmacy and the patient. Surrey, UK: MTP Press Ltd.; 1983:121-126. Print ISBN: 978-94-011-9725-0. Chapter 12.

6. Müller KH. Corticoid-Verdünnungen. Neue Entwicklungen in der Dermatologie. Band 5. Berlin, Heidelberg: Springer-Verlag; 1988:96-104.

7. McKenzie AW, Atkinson RM. Topical activities of betamethasone esters in man. Arch Dermatol 1964;89:741-746.

8. Boonsaner P, Remon JP, De Rudder D. The stability and blanching efficiency of some Betnelan- $V^{\circledR}$ cream dilutions. J Clin Hosp Pharm 1986;11:101-106.

9. Ryatt KS, Feather JW, Mehta A, et al. The effect of serial dilution of betamethasone-17-valerate on blanchng potential and chemical stability. J Clin Hosp Pharm 1983;8:143-145.
10. Woodford R. Investigation of the release characteristics of Unguentum Merck as a diluent for topical corticosteroid preparations. Curr Ther Res 1981;29:17-23.

11. Cornarakis-Lentzos M, Cowin PR. Dilutions of corticosteroid creams and ointments - a stability study. J Pharm Biomed Anal 1987;5:707-716.

12. Mehta AC, Calvert RT, Ryatt KS. Betamethasone 17-valerate: an investigation into its stability in Betnovate after dilution with emulsifying ointment: quantitation of degradation products. $\mathrm{Br} \mathrm{J}$ Pharm Pract 1982;4:10-13.

13. Byrne J, Velasco-Torrijos T, Reinhardt R. Development and validation of a novel stability-indicating HPLC method for the simultaneous assay of betamethasone-17-valerate, fusidic acid, potassium sorbate, methylparaben and propylparaben in a topical cream preparation. J Pharm Biomed Anal 2014;96: 111-117.

14. Byrne J, Velasco-Torrijos T, Reinhardt R. An RP-HPLC method for the stability-indicating analysis of impurities of both fusidic acid and betamethasone-17-valerate in a semi-solid pharmaceutical dosage form. J Chromatogr Sci 2015;53:1498-1503.

15. Note for guidance on manufacture of the finished dosage form (CPMP/QWP/486/95).

16. Specifications and control tests on the finished product, 3AQ11a, Directive 75/318/EEC, June 1992.

17. Bundgaard H, Hansen J. Studies on the stability of corticosteroids VI. Kinetics of the rearrangement of betamethasone-17-valerate to the 21-valerate ester in aqueous solution. Int J Pharm 1981;7: 197-203.

18. Yip YW, Li Wan Po A. Kinetics of decomposition and formulation of hydrocortisone butyrate in semiaqueous and gel systems. J Pharm Sci 1982;72:776-781.

19. Patent: WO/1996/040042. Stabilized steroid compositions

20. European Pharmacopeia: Monograph for Betamethasone valerate, 7.0/0811

21. Högger P. Critical formulation aspects of local corticosteroids. Eur J Hosp Pharm Pract 2011;17:60-61. 